\title{
The static potential: lattice versus perturbation theory in a renormalon-based approach
}

\author{
Antonio Pineda ${ }^{1}$ \\ Dept. d'Estructura i Constituents de la Matèria and IFAE, U. Barcelona \\ Diagonal 647, E-08028 Barcelona, Catalonia, Spain
}

\begin{abstract}
We compare, for the static potential and at short distances, perturbation theory with the results of lattice simulations. We show that a renormalon-dominance picture explains why in the literature sometimes agreement, and another disagreement, is found between lattice simulations and perturbation theory depending on the different implementations of the latter. We also show that, within a renormalon-based scheme, perturbation theory agrees with lattice simulations.
\end{abstract}

PACS numbers: 12.38.Bx, 12.38.Gc, 12.38.Cy.

\footnotetext{
${ }^{1}$ pineda@ecm.ub.es
} 


\section{Introduction}

The static potential is the object more accurately studied by (quenched) lattice simulations. This is due to its relevance in order to understand the dynamics of QCD. On the one hand, it is a necessary ingredient in a Schrödinger-like formulation of the Heavy Quarkonium. On the other hand, a linear growing behavior at long distance is signaled as a proof of confinement. Moreover, throughout the last years, lattice simulations $[1,2,3]$ have improved their predictions at short distances allowing, for the first time ever, the comparison between perturbation theory and lattice simulations. Therefore, the static potential provides a unique place where to test lattice and/or perturbation theory (depending on the view of the reader), as well as an ideal place where to study the interplay between perturbative and non-perturbative physics. This is even more so since the accuracy of the perturbative prediction of the static potential has also improved significantly recently $[4,5,6,7]$.

Let us first review the status of the art nowadays. The prediction for the perturbative static potential at two loops [4] indicated the failure (non-convergence) of perturbation theory at amazingly short distances. This failure of perturbation theory is not solved by the inclusion of the leading logs at three loops computed in $[5,6]$ nor by performing a renormalization group (RG) improvement of the static potential at next-to-next-to-leading log (NNLL) [7]. ${ }^{1}$

On the other hand, it was soon realized that the static potential suffered of renormalons [10] and that the leading one (of $O\left(\Lambda_{\mathrm{QCD}}\right)$ ) cancelled with the leading renormalon of twice the pole mass [11]. Nevertheless, the prediction of perturbation theory for the slope of the static potential should not suffer, in principle, from this renormalon and could be compared with lattice simulations, which, indeed, only predict the potential up to a constant. This comparison was performed in Ref. [12], where they compared the RG improved predictions (without including ultrasoft log resummation) of perturbation theory up to next-to-next-toleading order (NNLO) with lattice simulations. They indeed found that the discrepancies with lattice simulations and the lack of convergence of the perturbative series remained. They also found that the difference between perturbation theory and lattice could be parameterized by a linear potential in a certain range. Therefore, it seemed to support the claims of some groups $[13,14]$ of the possible existence of a linear potential at short distances. Such claims contradict the predictions of the operator product expansion (OPE), which state that the leading non-perturbative corrections are quadratic in distance at short distances ${ }^{2}$.

On the other hand, driven by the analysis of Refs. [15], it has been argued [16] that, within a renormalon based picture, perturbation theory agrees with the phenomenological potentials aimed to describe Heavy Quarkonium. For his study, he used the static version of the 1S mass and the upsilon expansion [17], which cancels the leading renormalon and relate the $1 \mathrm{~S}$ mass to the $\overline{\mathrm{MS}}$ mass. Moreover, In Ref. [18] (see also [16, 19]), it has been shown that perturbation theory can indeed reproduce the slope of the static potential given

\footnotetext{
${ }^{1}$ There also exists a computation of the running of the Coulomb potential in vNRQCD [8] with the same precision that disagrees with the one obtained in pNRQCD [7]. At this respect, we would like to report on a recent computation [9] of the 4-loop double $\log$ term of the Coulomb potential proportional to $C_{A}^{3} \beta_{0}$ that agrees with the pNRQCD result and disagrees with the vNRQCD one.

${ }^{2}$ This contradiction is indeed so only if one believes that perturbation theory can be applied for the shortest distances available in lattice simulations $\sim 1-4 \mathrm{GeV}$. We thank V.I. Zakharov for stressing this point to us.
} 
by lattice simulations at short distances by using the force instead of the potential as the basic tool without the need to talk about renormalons.

In this paper, we would like to try to clarify further the above issues and support the renormalon dominance picture by comparing the potential computed in lattice with the RS static potential (a renormalon free definition of the potential obtained in Ref. [20]).

\section{On-shell scheme}

The energy of two static sources in a singlet configuration reads (in the on-shell (OS) scheme)

$$
E(r)=2 m_{\mathrm{OS}}+\lim _{T \rightarrow \infty} \frac{i}{T} \ln \left\langle W_{\square}\right\rangle .
$$

In the situation $\Lambda_{\mathrm{QCD}} \ll 1 / r$, it can be computed order by order in $\alpha_{\mathrm{s}}(\nu)(\nu \sim 1 / r)$ and in the multipole expansion (see $[21,7]$ )

$$
E(r)=2 m_{\mathrm{OS}}+V_{s, \mathrm{OS}}\left(r, \nu_{\mathrm{US}}\right)+\delta E_{\mathrm{US}}\left(r, \nu_{\mathrm{US}}\right) .
$$

$m_{\mathrm{OS}}$ is the pole mass. The static potential reads $\left(\alpha_{\mathrm{s}} \equiv \alpha_{\mathrm{s}}(\nu)\right)$

$$
V_{s, \mathrm{OS}} \equiv V_{s, \mathrm{OS}}\left(r, \nu_{\mathrm{US}}\right)=\sum_{n=0}^{\infty} V_{n} \alpha_{\mathrm{s}}^{n+1} \text {, }
$$

where $V_{n} \equiv V_{n}\left(r, \nu, \nu_{\mathrm{US}}\right)$. The first three coefficients $V_{0}, V_{1}$ and $V_{2}$ are known [4]. The log-dependence on $\nu$ of $V_{3}$ can also be obtained by using the $\nu$-independence of $V_{s, \text { Os }}$. The log-dependence on $\nu_{\mathrm{US}}$ of $V_{3}$ is also known [5]. Therefore, the only unknown piece of $V_{3}$ is a $\nu / \nu_{\mathrm{US}}$-independent constant. Its size has been estimated in Ref. [20] assuming renormalon dominance. For $n_{f}=0$, it reads

$$
V_{3}(r, 1 / r, 1 / r)=1 / r \times(-76.1075)
$$

Leaving aside the leading-renormalon independent contributions to $V_{3}$, this number suffers from two sources of errors (see Ref. [20] for details). One is the error in the evaluation of the normalization factor of the renormalon, $N_{m}$, which we will discuss in the next section. The other error is due to $O(1 / n)$ corrections ( $n=3$ in this case), which appear to be negligible. The relative $O(1 / n)$ corrections are -0.0264977 , and the $O\left(1 / n^{2}\right)$ corrections are -0.00544012 . Therefore, we expect the unknown $O\left(1 / n^{3}\right)$ effects to be very small. We will use Eq. (4) in the following for our estimates of $V_{3}$.

It is quite remarkable that the numbers obtained in Ref. [20] for $V_{3}$ are quite close to the ones obtained in Ref. [22] using Padé-approximants methods. This is even so if we do the Fourier transform back to momentum space. We display in Table 1 the numbers one obtains from Ref. [20] for the coefficient $c_{0}$ (the portion of the three-loop momentum space coefficient of the static potential independent of scale logarithms as defined in Ref. [22]) for different number of massless flavours. We can see that the difference with Chishtie and Elias results (see table 1 in Ref. [22]) is always of the order of 20-30 and roughly independent of 


\begin{tabular}{|c||c|c|c|c|c|c|c|}
\hline$n_{f}$ & 0 & 1 & 2 & 3 & 4 & 5 & 6 \\
\hline$c_{0}$ & 292 & 227 & 168 & 116 & 72 & 37 & 12 \\
\hline
\end{tabular}

Table 1: Values of $c_{0}$, as defined in Ref. [22], according to the results of Ref. [20].

the number of flavours. Therefore for $n_{f} \rightarrow 0$, where the absolute value of $c_{0}$ increases, the bulk of $c_{0}$ agrees with Chishtie and Elias prediction.

The static potential is $\nu$-independent. By setting $\nu=1 / r$, we could effectively resum the $\ln \nu r$ terms. The RG-improved expressions would read

$$
V_{s, \mathrm{OS}}=\sum_{n=0}^{\infty} V_{n}\left(r, 1 / r, \nu_{\mathrm{US}}\right) \alpha_{\mathrm{s}}(1 / r)^{n+1},
$$

and we would have expressions for $n=0,1,2,3$. In the above expression we have considered $\ln r \nu_{\mathrm{US}} \sim 1$. Since $\ln r \nu_{\mathrm{US}} \gg 1$ for some range of the parameters, one could be in the situation where one also has to resum ultrasoft logs as it has been done in Ref. [7]. Nevertheless, explicit calculations show that, at least for the range of parameters studied in this paper, higher order ultrasoft logs are subleading even if sizable. However, for definiteness, we will work with the resummed expression (the physical picture does not change anyhow), which we will add to $V_{3}(r, 1 / r, 1 / r)$ :

$$
V_{3}\left(r, 1 / r, \nu_{\mathrm{US}}\right) \equiv V_{3}(r, 1 / r, 1 / r)+\frac{C_{A}^{3}}{6 \beta_{0}} \alpha_{\mathrm{S}}^{3}\left(r^{-1}\right) \log \left(\frac{\alpha_{s}\left(r^{-1}\right)}{\alpha_{s}\left(\nu_{u s}\right)}\right) .
$$

In principle, it could be considered to be more consistent to add the resummed expression for the ultrasoft $\operatorname{logs}$ to $V_{2}$. Nevertheless, we will add them to $V_{3}$ since it is the counting consistent when the leading single log gives the bulk of the correction (moreover, we will see that, even after the leading-renormalon cancellation is achieved, its effect is small ${ }^{3}$ if compared with the typical estimate of the terms of $V_{3}$ ).

The leading $O\left(r^{2}\right)$ non-vanishing contribution to $\delta E_{\mathrm{US}}\left(r, \nu_{\mathrm{US}}\right)$ reads (we actually write the Euclidean expression)

$$
\delta E_{\mathrm{US}}\left(r, \nu_{u s}\right) \simeq \frac{T_{F}}{3 N_{c}} \mathbf{r}^{2} \int_{0}^{\infty} d t e^{-t\left(V_{o}, \mathrm{OS}-V_{s, \mathrm{OS}}\right)}\left\langle g \mathbf{E}^{a}(t) \phi(t, 0)_{a b}^{\mathrm{adj}} g \mathbf{E}^{b}(0)\right\rangle\left(\nu_{u s}\right)
$$

At the order of interest $V_{o, \mathrm{OS}} \equiv \frac{1}{2 N_{c}} \frac{\alpha_{\mathrm{s}}}{r}$ and $V_{s, \mathrm{OS}}$ is approximated to its leading order value: $-C_{F} \frac{\alpha_{\mathrm{s}}}{r}$. This contribution cancels the leading $\nu_{\mathrm{US}}$ scale dependence of $V_{s, \mathrm{OS}}$. For the scales we will study in this paper (those accessible by lattice simulations), we will consider $\alpha_{\mathrm{s}} / r$ to be a non-perturbative scale. Therefore, Eq. (7) can not be computed perturbatively and it will not be considered in this and the following section, where we aim to a pure perturbative prediction (it will be considered, however, in sec. 4.1). Thus, our results will depend on $\nu_{u s}$, for which we will take $\nu_{u s}=2.5 r_{0}^{-1}$. We will set $n_{f}=0$ and work in lattice units: $r_{0}^{-1}$ (with $r_{0}^{-1} \simeq 400 \mathrm{MeV}$ according to lattice simulations), $\Lambda_{\overline{\mathrm{MS}}}=0.602(48) r_{0}^{-1}$ [23], since our aim will be to compare with lattice simulations. 


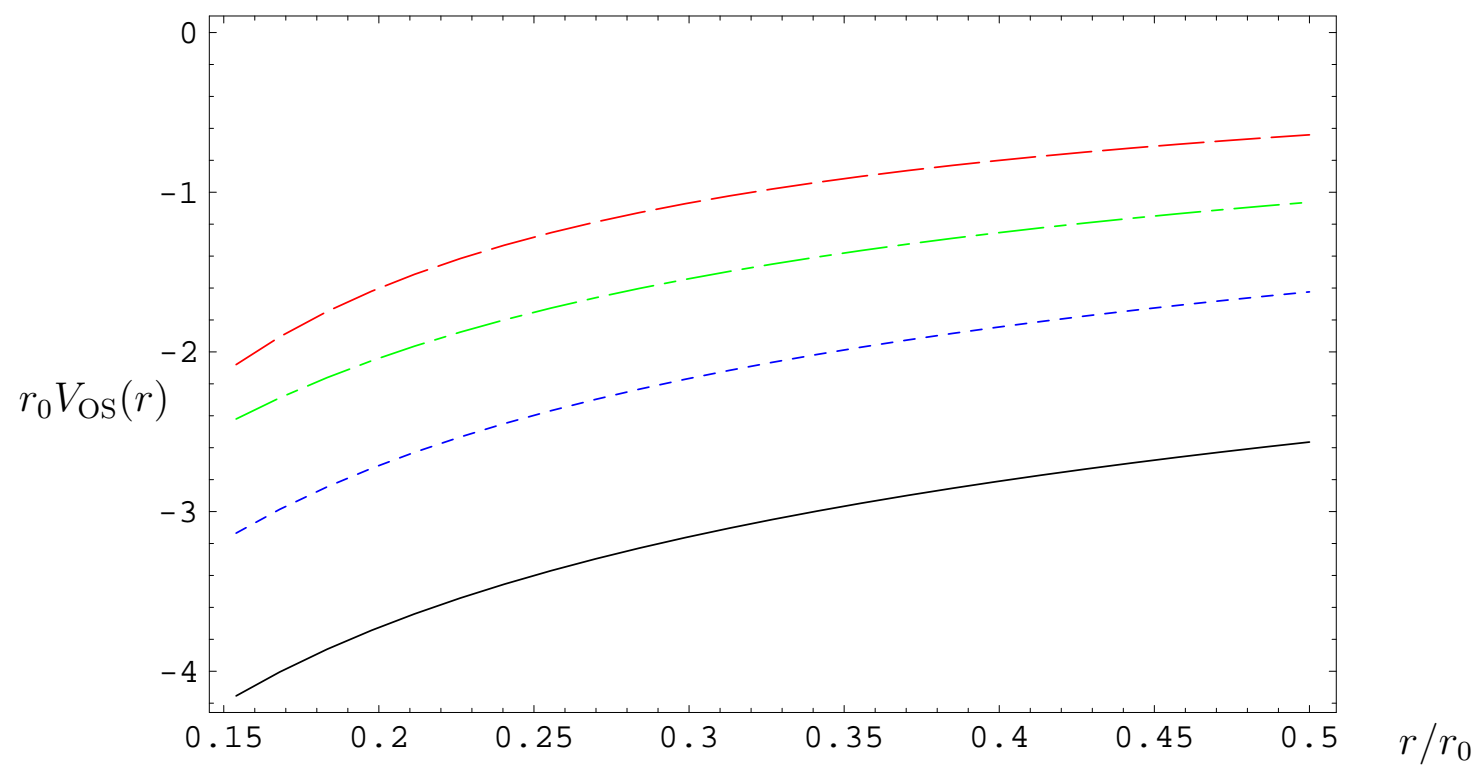

Figure 1: Plot of $r_{0} V_{\mathrm{OS}}(r)$ at tree (dashed line), one-loop (dash-dotted line), two-loops (dotted line) and three loops (estimate) plus the leading single ultrasoft log (solid line). For the scale of $\alpha_{\mathrm{s}}(\nu)$, we set $\nu=1 / 0.15399 r_{0}^{-1}$. $\nu_{u s}=2.5 r_{0}^{-1}$.

Now, we want to check the convergence of the series within perturbation theory. We first consider the perturbative static potential without trying to resum $\ln r \nu \operatorname{logs}$, ie. for a fixed scale $\nu$ for $\alpha_{\mathrm{s}}(\nu)$ (Eq. (3)). We show our results in Fig. 1, where we have chosen $\nu=1 / 0.15399 r_{0}^{-1}$. We see that it does not converge to a value. We next try with $\ln r \nu$ resummation (Eq. (5)), since these logs could be large. We show our results in Fig. 2. We find the same problem and moreover the slope appears to be different in both cases. The situation appears to be puzzling.

Let us now compare with lattice simulations. These predict the energy of an static quark-antiquark system up to an $r$-independent constant $^{4}$ :

$$
E_{\text {latt. }}(r)=k_{\text {latt. }}+\lim _{T \rightarrow \infty} \frac{i}{T} \ln \left\langle W_{\square}\right\rangle .
$$

Therefore, the slope of the potential is a physical prediction of the lattice static potential and does not suffer from the leading renormalon. The constant is usually fixed in a way such that

$$
E_{\text {latt. }}\left(r_{0}\right)=0 \text {, }
$$

except in Ref. [2] where $E_{\text {latt. }}\left(r_{c}\right)=0$ is used (for the definition of $r_{c}$ see [2]).

In order to compare the perturbative predictions with lattice simulations, we add a constant to the perturbative potential to fix both potentials to be equal at some scale $r^{\prime}$ :

$$
E_{\text {latt. }}\left(r^{\prime}\right)=k_{\text {per. }}\left(r^{\prime}\right)+V_{s, O S}\left(r^{\prime}\right) .
$$

\footnotetext{
${ }^{3}$ However, it remains to be seen whether, once $V_{3}$ is exactly computed and the $O\left(r^{2}\right)$ renormalon were also subtracted, the ultrasoft logs would be a subleading effect compared with finite pieces.

${ }^{4}$ Therefore, due to the unknown constant it can not be related with the underlying theory (QCD) and, for instance, obtain the mass.
} 


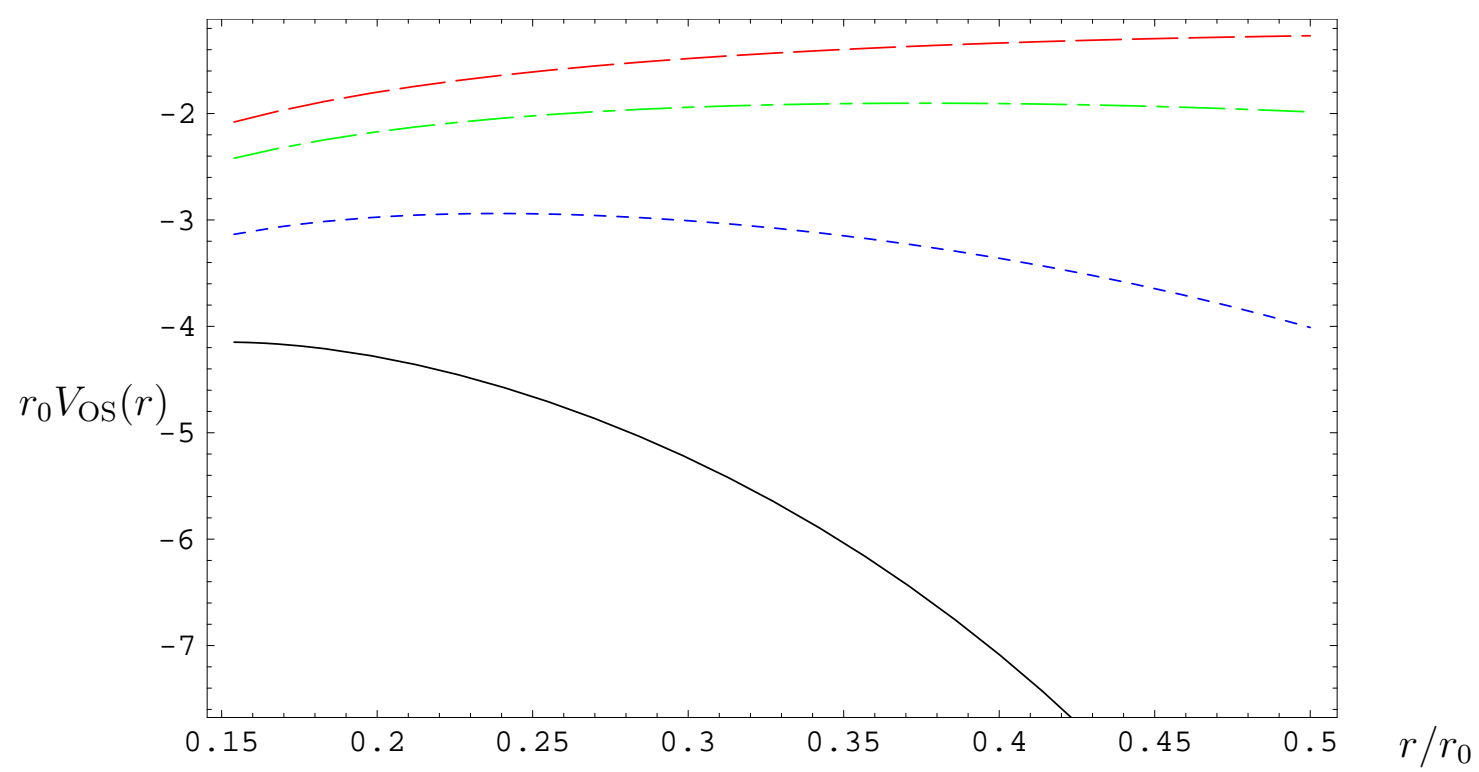

Figure 2: Plot of $r_{0} V_{\mathrm{OS}}(r)$ at tree (dashed line), one-loop (dash-dotted line), two-loops (dotted line) and three loops (estimate) plus the $R G$ expression for the ultrasoft logs (solid line). For the scale of $\alpha_{\mathrm{s}}(\nu)$, we set $\nu=1 / r$. $\nu_{u s}=2.5 r_{0}^{-1}$.

The value of this constant varies according to the order the perturbative computation has been done, ie.

$$
k_{\text {pert. }}\left(r^{\prime}\right)=k_{0}\left(r^{\prime}\right)+k_{1} \alpha_{\mathrm{s}}\left(r^{\prime}\right)+k_{2} \alpha_{\mathrm{s}}^{2}\left(r^{\prime}\right)+\cdots .
$$

We will consider the lattice data of Refs. [1,2]. They both appear to be perfectly compatible with each other for finite lattice spacings. In any case, the lattice data of Ref. [2], being more recent, appears to have smaller errors with a very small spread of the points around the fitted curve. Here, in order to get the highest possible precision, we will use the lattice data of Ref. [2] for which the continuum limit has been reached. For comparison with the lattice points at finite lattice spacing of Ref. [1] and for a somewhat a larger range, we refer to the 1st version of this paper sent to the web. The physical outcome is, in any case, the same.

The comparison with lattice is performed in Fig. 3 for the perturbative result without $\ln (r \nu)$ resummation ( $\nu$ constant) and in Fig. 4 for the perturbative result with $\ln (r \nu)$ resummation $(\nu=1 / r)$. As explained above, we have added a constant to the perturbative potential (different at each order) as to make it equal to the lattice potential at $r=0.15399 r_{0}$. We see a completely different behavior in both cases. Whereas for the latter, the agreement goes worse as we increase the order of our calculation, for the former the agreement with lattice improves ${ }^{5}$. Indeed, it is quite remarkable that lattice simulations are precise enough to see the log behavior of $\alpha_{\mathrm{s}}$ predicted by asymptotic freedom.

In the next section we will explain the above behavior (why sometimes perturbation theory can describe lattice data and some others not) within an controlled framework where the renormalon is taken into account.

\footnotetext{
${ }^{5}$ Usually, the comparison between lattice and perturbation theory was performed using the expressions with $\nu=1 / r$, whereas the analysis with $\nu$ constant was largely unnoticed.
} 


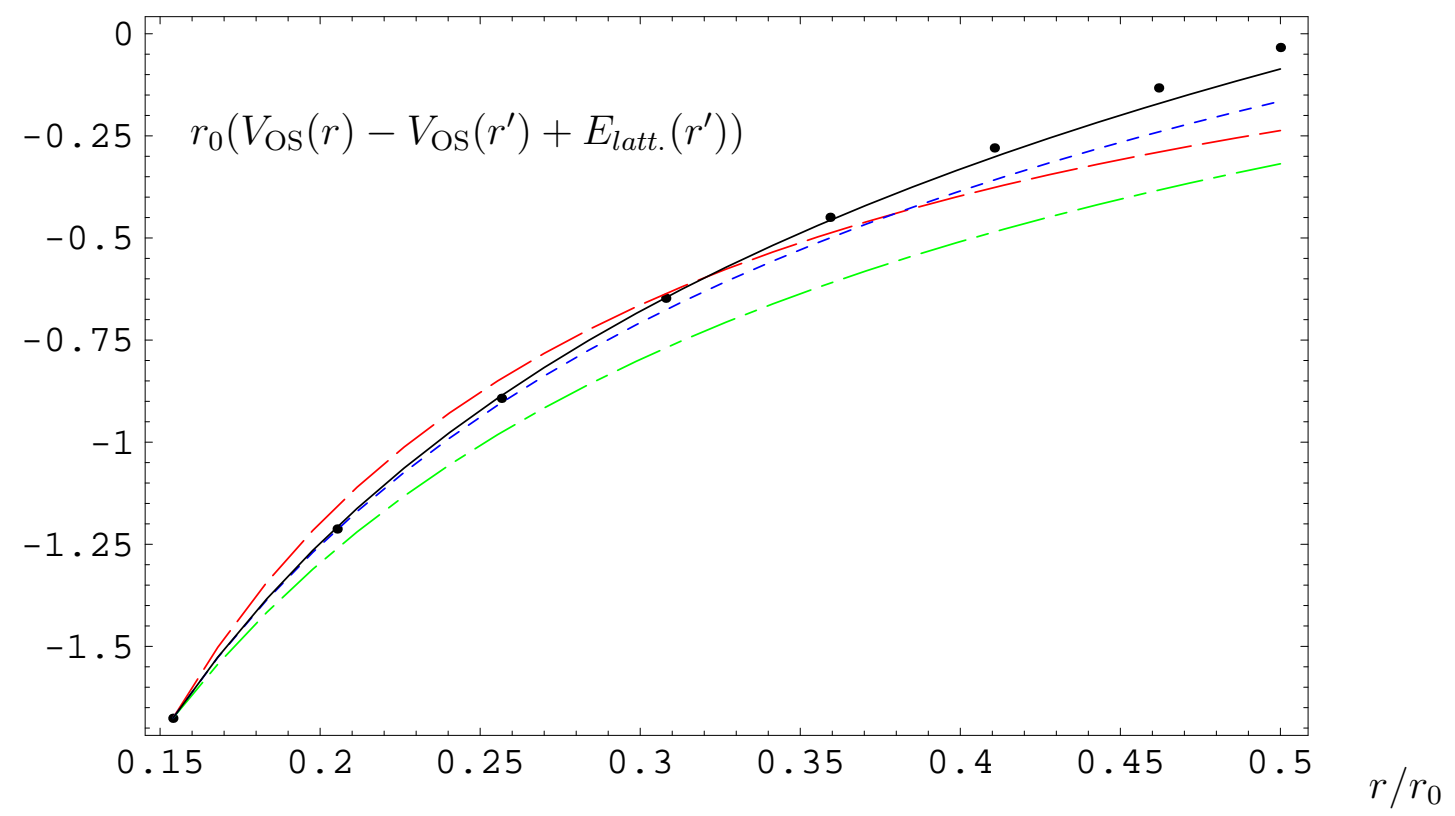

Figure 3: Plot of $r_{0}\left(V_{\mathrm{OS}}(r)-V_{\mathrm{OS}}\left(r^{\prime}\right)+E_{\text {latt. }}\left(r^{\prime}\right)\right)$ versus $r$ at tree (dashed line), one-loop (dash-dotted line), two-loops (dotted line) and three loops (estimate) plus the leading single ultrasoft log (solid line) compared with the lattice simulations [2] $E_{\text {latt. }}(r)$. For the scale of $\alpha_{\mathrm{s}}(\nu)$, we set $\nu=1 / 0.15399 r_{0}^{-1}$. $\nu_{u s}=2.5 r_{0}^{-1}$ and $r^{\prime}=0.15399 r_{0}$.

\section{RS scheme}

The RS scheme was defined in Ref. [20] aiming to eliminate the renormalons of the matching coefficients appearing in Heavy Quarkonium calculations. In particular, the leading renormalon of the heavy quark mass and the static potential. We refer to Ref. [20] for details. Here, we just write the relevant formulas needed for our analysis.

Analogously to the OS scheme, the energy of two static sources in a singlet configuration reads

$$
E_{s}(r)=2 m_{\mathrm{RS}}\left(\nu_{f}\right)+\left(\lim _{T \rightarrow \infty} \frac{i}{T} \ln \left\langle W_{\square}\right\rangle-2 \delta m_{\mathrm{RS}}\left(\nu_{f}\right)\right)
$$

where ${ }^{6}$

$$
\delta m_{\mathrm{RS}}\left(\nu_{f}\right)=\sum_{n=1}^{\infty} \delta m_{\mathrm{RS}, \mathrm{n}} \alpha_{\mathrm{s}}^{n+1}=\sum_{n=1}^{\infty} N_{m} \nu_{f}\left(\frac{\beta_{0}}{2 \pi}\right)^{n} \alpha_{\mathrm{s}}^{n+1}\left(\nu_{f}\right) \sum_{k=0}^{\infty} c_{k} \frac{\Gamma(n+1+b-k)}{\Gamma(1+b-k)},
$$

and $m_{\mathrm{RS}} \equiv m_{\mathrm{OS}}-\delta m_{\mathrm{RS}}$. If the beta function were known to infinity order in perturbation theory, it would be possible to obtain all the coefficients $b$ and $c_{k}$ [24]. In practice, only $b$ and $c_{0,1,2}$ are known (see $[24,20,25]$ ). For $N_{m}$ only an approximate calculation is possible using some ideas first developed in Ref. [26]. This computation has been done in Ref. [20].

\footnotetext{
${ }^{6}$ Actually, we are going to use in this paper the RS' scheme defined in Ref. [20] instead of the RS scheme, since we believe it has a more physical interpretation. Nevertheless, we will denote it in this paper as RS scheme in order to simplify the notation. In any case, the physical picture does not change. The results would also converge towards the result predicted by lattice simulations but with a different pattern.
} 


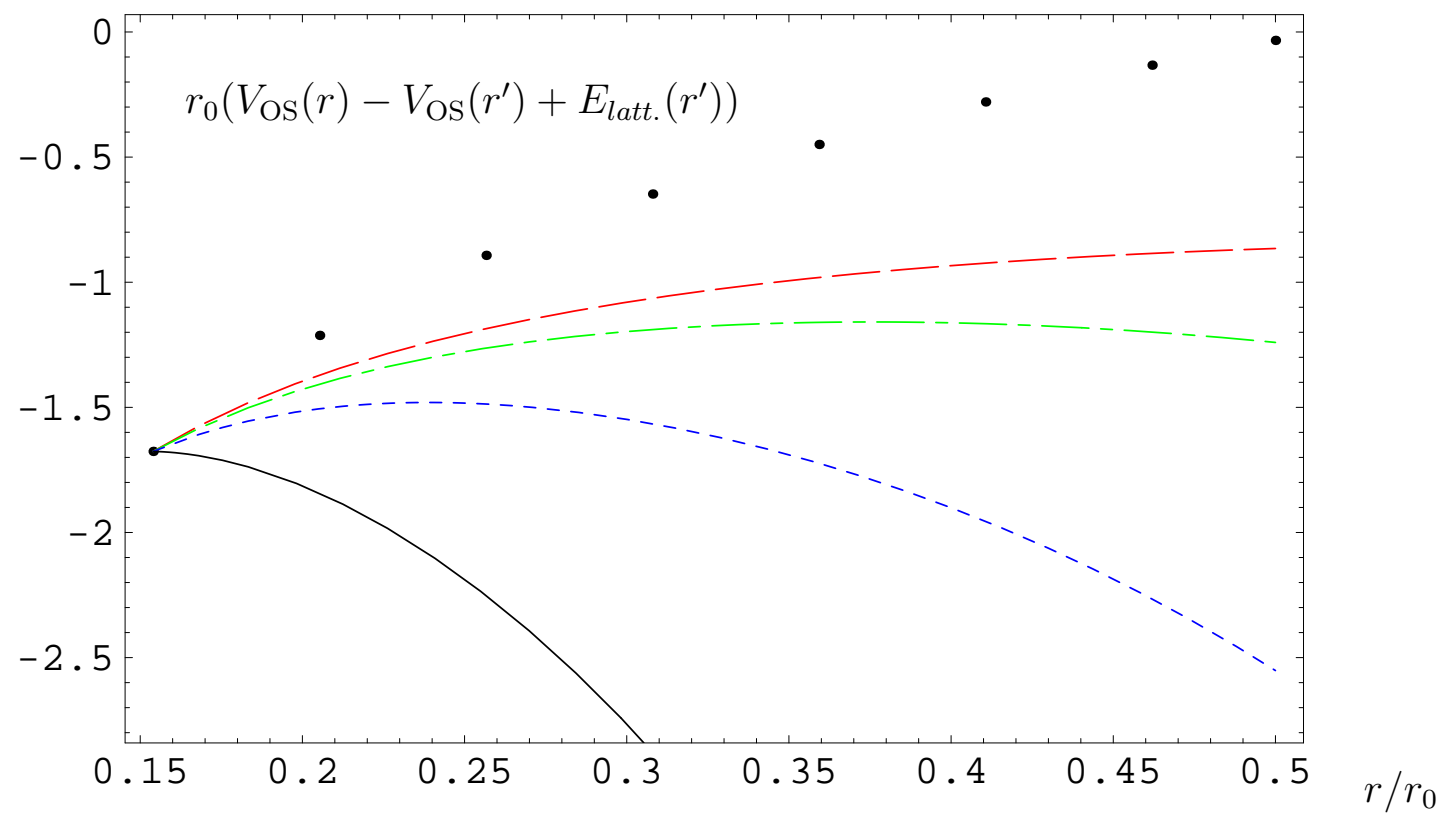

Figure 4: Plot of $r_{0}\left(V_{\mathrm{OS}}(r)-V_{\mathrm{OS}}\left(r^{\prime}\right)+E_{\text {latt. }}\left(r^{\prime}\right)\right)$ versus $r$ at tree (dashed line), one-loop (dash-dotted line), two-loops (dotted line) and three loops (estimate) plus the RG expression for the ultrasoft logs (solid line) compared with the lattice simulations [2] $E_{\text {latt. }}(r)$. For the scale of $\alpha_{\mathrm{s}}(\nu)$, we set $\nu=1 / r$. $\nu_{u s}=2.5 r_{0}^{-1}$ and $r^{\prime}=0.15399 r_{0}$.

The result reads (for $n_{f}=0$ )

$$
N_{m}=0.424413+0.174732+0.0228905=0.622036,
$$

where each term corresponds to a different power in $u$ (in the Borel plane) of the calculation (see Ref. [20] for details). We see a nice convergence. This number will be the one we will use in the following. This number should be equal to $-2 N_{V}$, where $N_{V}$ is the normalization factor of the renormalon of the static potential. $N_{V}$ was also approximately computed in Ref. [20]. For $n_{f}=0$, it reads

$$
N_{V}=-1.33333+0.499433-0.338437=-1.17234 .
$$

In this case the convergence is not so good but we have an alternating series. In any case, we see that both values appear to be quite close:

$$
2 \frac{2 N_{m}+N_{V}}{2 N_{m}-N_{V}}=0.059 .
$$

We take this as an approximate indication of the error in the evaluation of $N_{m}$.

In the situation $\Lambda_{\mathrm{QCD}} \ll 1 / r, E_{s}(r)$ can be factorized in the following way (with the accuracy we are working, the expression for $\delta E_{s, \mathrm{US}}$ will be equal in the OS and RS scheme):

$$
E_{s}(r)=2 m_{\mathrm{RS}}\left(\nu_{f}\right)+V_{s, \mathrm{RS}}\left(r ; \nu_{u s} ; \nu_{f}\right)+\delta E_{s, \mathrm{US}}\left(r, \nu_{u s}\right) .
$$


$V_{s, \mathrm{RS}}(r) \equiv V_{s, \mathrm{RS}}\left(r ; \nu_{u s} ; \nu_{f}\right)$ was defined in Ref. [20] (note that we expand $V_{s}$ and $\delta m_{\mathrm{RS}}$ with the same $\alpha_{\mathrm{s}}$ ):

$$
V_{s, \mathrm{RS}}(r)=V_{s}+2 \delta m_{\mathrm{RS}}=\sum_{n=1}^{\infty}\left(V_{n}+2 \delta m_{\mathrm{RS}, \mathrm{n}}\right) \alpha_{\mathrm{s}}^{n}=\sum_{n=0}^{\infty} V_{\mathrm{RS}, \mathrm{n}}^{(0)} \alpha_{\mathrm{s}}^{n+1} .
$$

Due to the leading renormalon, the behavior of the perturbative expansion at large orders of $V_{n}$ reads [24, 20]

$$
V_{n} \stackrel{n \rightarrow \infty}{=} N_{V} \nu\left(\frac{\beta_{0}}{2 \pi}\right)^{n} \frac{\Gamma(n+1+b)}{\Gamma(1+b)}\left(1+\frac{b}{(n+b)} c_{1}+\frac{b(b-1)}{(n+b)(n+b-1)} c_{2}+\cdots\right) .
$$

Therefore, by adding $\delta m_{\mathrm{RS}}$ to $V_{s}$ we expect to cancel the renormalon of the static potential. Indeed, what we want is to obtain the renormalon cancellation order by order in perturbation theory. This means to kill the $O(n !)$ behavior of the coefficient multiplying the $O\left(\alpha_{\mathrm{s}}^{n}\right)$ term of $V_{s}$ with the $O(n !)$ behavior that appears in $\delta m_{\mathrm{RS}}$ such that $V_{\mathrm{RS}}$ enjoys nicer convergence properties. In principle, this cancellation should hold for an arbitrary $\nu$ and, in particular, for $\nu=1 / r$. This, indeed, is the key observation of this paper and it will become relevant later on in order to explain the results in the previous section as well as in the literature.

In principle, any $\nu_{f}$ should cancel the renormalon of the static potential (we have the constraint, though, that $\nu_{f}$ should be large enough to fulfill $\left.\alpha_{\mathrm{s}}\left(\nu_{f}\right) \ll 1\right)$. If we re-expand $\delta m_{\mathrm{RS}}\left(\nu_{f}\right)$ in terms of $\alpha_{\mathrm{s}}(\nu)$, the renormalon is still kept in $\delta m_{\mathrm{RS}}\left(\nu_{f}\right)$ but new terms are generated, which, nevertheless, should not belong to the renormalon. In other words

$$
\delta m_{\mathrm{RS}}\left(\nu_{f}\right)=\delta m_{\mathrm{RS}}(\nu)+F\left(\nu ; \nu_{f}\right),
$$

where the Borel transform of $F\left(\nu ; \nu_{f}\right)$ should be analytic at $u=1 / 2$ in the Borel plane. This can be easily seen in the large $\beta_{0}$ limit. In more formal terms what we have is

$$
\Lambda_{\overline{\mathrm{MS}}}=\text { constant } \operatorname{Im}\left[\delta m_{\mathrm{RS}}\left(\nu_{f}\right)\right], \quad \Lambda_{\overline{\mathrm{MS}}}=\text { constant } \operatorname{Im}\left[\delta m_{\mathrm{RS}}(\nu)\right]
$$

with the same constant by definition (indeed this is what defines the renormalon). Therefore, the conclusion is that

$$
\operatorname{Im}\left[F\left(\nu ; \nu_{f}\right)\right]=0
$$

to the order of interest, concluding that $F$ does not have the closest singularity in the Borel plane. Note that in order to have the structure of Eq. (20) is necessary to have the complete renormalon contribution, ie. to all orders in perturbation theory. If we have the renormalon to some given order in perturbation theory, a change of the scale will produce large (renormalon-related) terms that would not cancel since we do not have the complete sum. Indeed, this is the explanation why in fixed order perturbation theory one has to expand on $\alpha_{\mathrm{s}}$ at the very same scale for the renormalon and the OS result in order to achieve the renormalon cancellation order by order in $\alpha_{\mathrm{s}}$.

On the other hand, $\nu_{f}$ can not be arbitrarily large. Otherwise the power counting is broken. This restricts $\nu_{f} \lesssim 1 / r$ in order to ensure the counting $V_{\mathrm{RS}} \sim O\left(\alpha_{\mathrm{s}} / r\right)$. In our case, we will set $\nu_{f}=2.5 r_{0}^{-1} \sim 1 \mathrm{GeV}$. In any case, when studying the slope of the potential, the result should be independent on $\nu_{f}$, as $\delta m_{\mathrm{RS}}\left(\nu_{f}\right)$ is just an r-independent constant. At this 
respect, we should say that this is indeed so to a large extent. If we take $\nu_{f}=5 r_{0}^{-1} \sim 2 \mathrm{GeV}$ agreement with lattice is also obtained ${ }^{7}$. One could also think of setting $\nu_{f}=1 / r$. This would mean to subtract an $r$-dependent constant to the static potential. This is not what it is done in lattice simulations since they are arbitrary up to a $r$-independent constant. Therefore, this would not agree with lattice simulations.

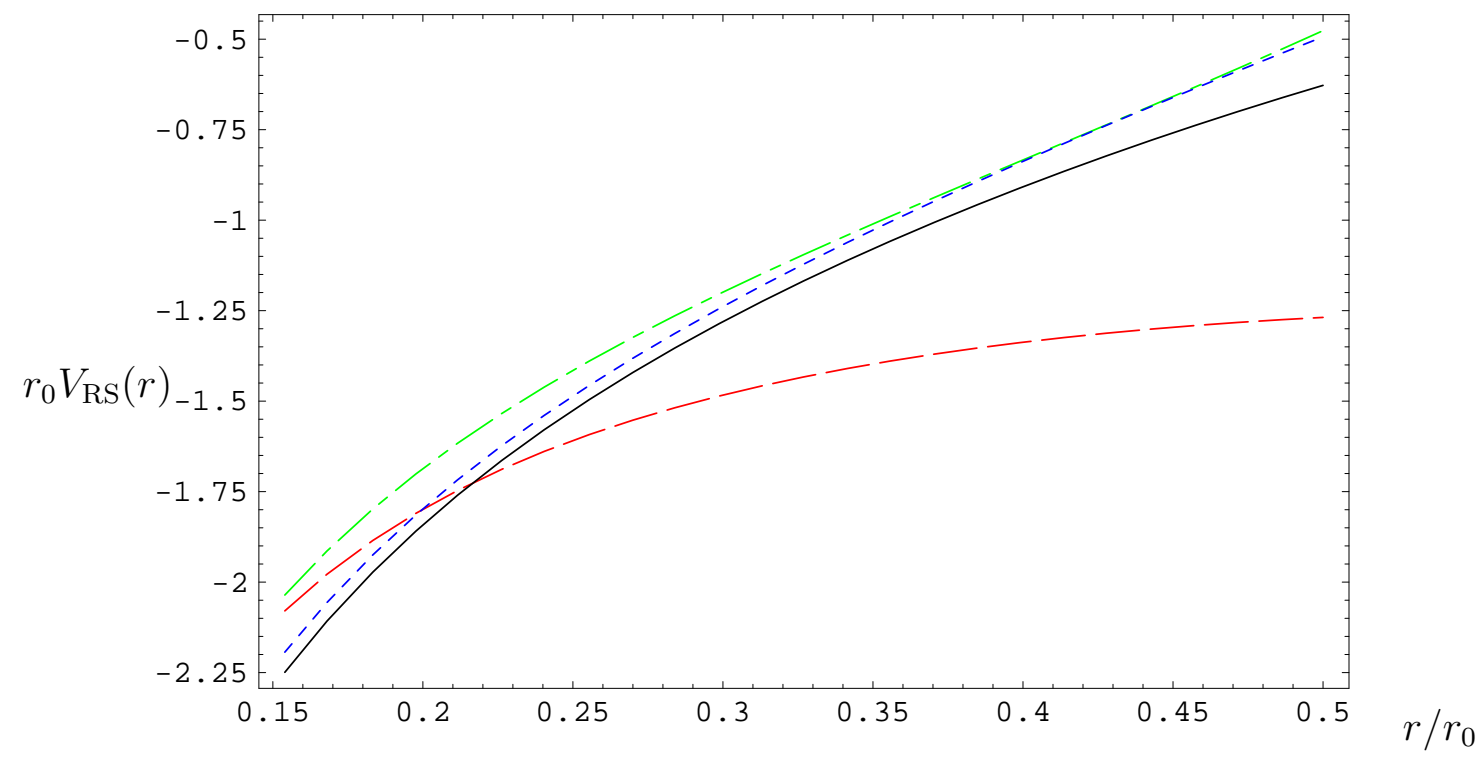

Figure 5: Plot of $r_{0} V_{\mathrm{RS}}(r)$ at tree (dashed line), one-loop (dash-dotted line), two-loops (dotted line) and three loops (estimate) plus the $R G$ expression for the ultrasoft logs (solid line). For the scale of $\alpha_{\mathrm{s}}(\nu)$, we set $\nu=1 / r$. $\nu_{u s}=2.5 r_{0}^{-1}$ and $\nu_{f}=2.5 r_{0}^{-1}$.

In conclusion, by using $V_{\mathrm{RS}}$, we expect the bad perturbative behavior due to the renormalon to disappear. Let us see that this is indeed so. Working analogously to the OS scheme, we consider the RS potential at different orders in perturbation theory with $\ln (r \nu)$ resummation or not. We display our results in Figs. 5 and 6 . In the first case, the $\ln (r \nu)$ resummation is achieved by setting $\nu=1 / r$ and the ultrasoft log resummation is also included. In the second case, we set $\nu=1 / 0.15399 r_{0}^{-1}$ and only the leading single ultrasoft $\log$ is included. We see that now we do not have the gap between different orders in the perturbative expansion (or it is dramatically reduced). Moreover, we do not have a (dramatically) different behavior of the slope of the potential; $\ln (r \nu)$ resummation or not converges to the same value.

We can now compare with lattice simulations: Figs. 3 and 7. We work analogously to the OS scheme. Now, the constant we have to add is approximately $\alpha_{\mathrm{s}}$-independent, reflecting that we have accurately achieved the renormalon cancellation. We see that our results are convergent to the same potential, which, as we can see, corresponds to the lattice potential.

\footnotetext{
${ }^{7}$ One could say that since $\delta m_{\mathrm{RS}}$ starts at $O\left(\alpha_{\mathrm{s}}^{2}\right)$ a larger value of $\nu_{f}$ could be used and yet not to break the power counting. Nevertheless, we refrain from using $\nu_{f} \sim 5 r_{0}^{-1}$ since we do not understand the meaning of the results with values of $\nu_{f}$ larger than $1 / r$. In particular, this would have a difficult explanation from an effective theory point of view, where $\nu_{f}$ is understood as an cutoff of the effective theory, which has to be smaller than $1 / r$.
} 


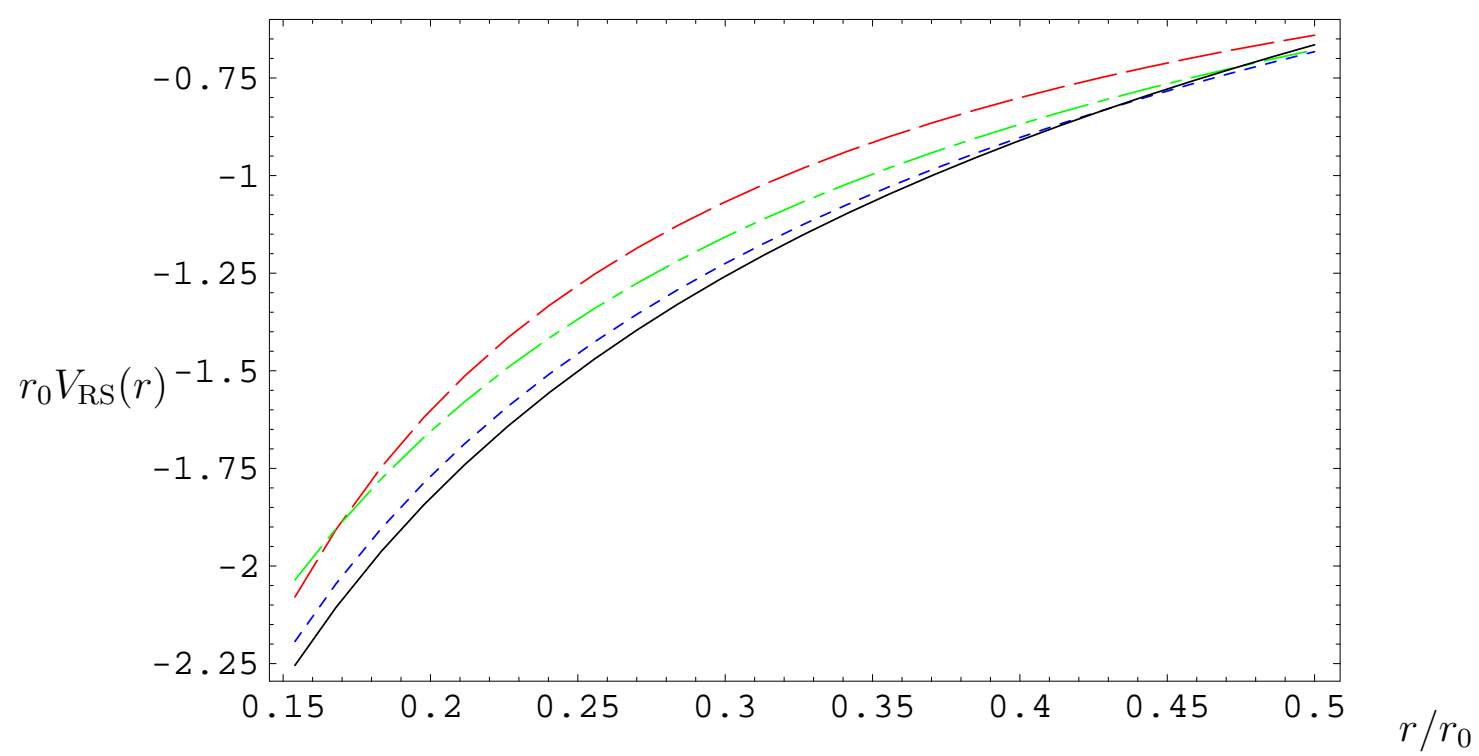

Figure 6: Plot of $r_{0} V_{\mathrm{RS}}(r)$ at tree (dashed line), one-loop (dash-dotted line), two-loops (dotted line) and three loops (estimate) plus the leading single ultrasoft log (solid line). For the scale of $\alpha_{\mathrm{s}}(\nu)$, we set $\nu=1 / 0.15399 r_{0}^{-1} . \nu_{u s}=2.5 r_{0}^{-1}$ and $\nu_{f}=2.5 r_{0}^{-1}$.

The case without $\ln (r \nu)$ resummation is exactly equal to the OS case, ie. to Fig. 3, since it is just equivalent to add a r-independent constant at each order in $\alpha_{\mathrm{s}}$. This explains, within the renormalon dominance picture, the success of that specific OS scheme calculation; it is just equivalent to an specific case of the renormalon based calculation: $\nu=$ constant. Therefore, perturbation theory without log resummation can explain lattice data up to $r \sim 0.5 r_{0}$ by subtracting a r-independent constant at each order in perturbation theory. This fact is understandable if the factorization scale chosen is not very far of $1 / r$ so that the RG does not play a decisive role plus the fact that subtracting a constant kills the renormalon. However, now, we are not restricted to take $\nu=$ constant but we can take $\nu=1 / r$, which allows us to resum the $\ln (r \nu)$ terms and yet cancel the renormalon as we can nicely see in Fig. 7.

Within the renormalon dominance picture, we can also explain why perturbation theory with $\ln (r \nu)$ resummation could not explain lattice data (see Fig. 4) even if subtracting a rindependent constant at each order in perturbation theory. In particular, this would explain the disagreement found in Ref. [12]. The explanation of this fact is that $\ln (r \nu)$ resummation is equivalent to set $\alpha_{\mathrm{s}}(r)$ as the expansion parameter. Therefore, the expansion parameter is a function of $r$ and, consequently, the amount to be subtracted at each order in $\alpha_{\mathrm{s}}(r)$ in order to cancel the renormalon is also $r$-dependent. It follows that the (perturbative) renormalon cancellation can not be achieved by only subtracting a $r$-independent constant at each order in $\alpha_{\mathrm{s}}(r)$ as it was done in Fig. 4.

We would like now to understand the agreement between lattice [18] (or potential models [16]) and perturbation theory if the static potential is reconstructed from the force in the 


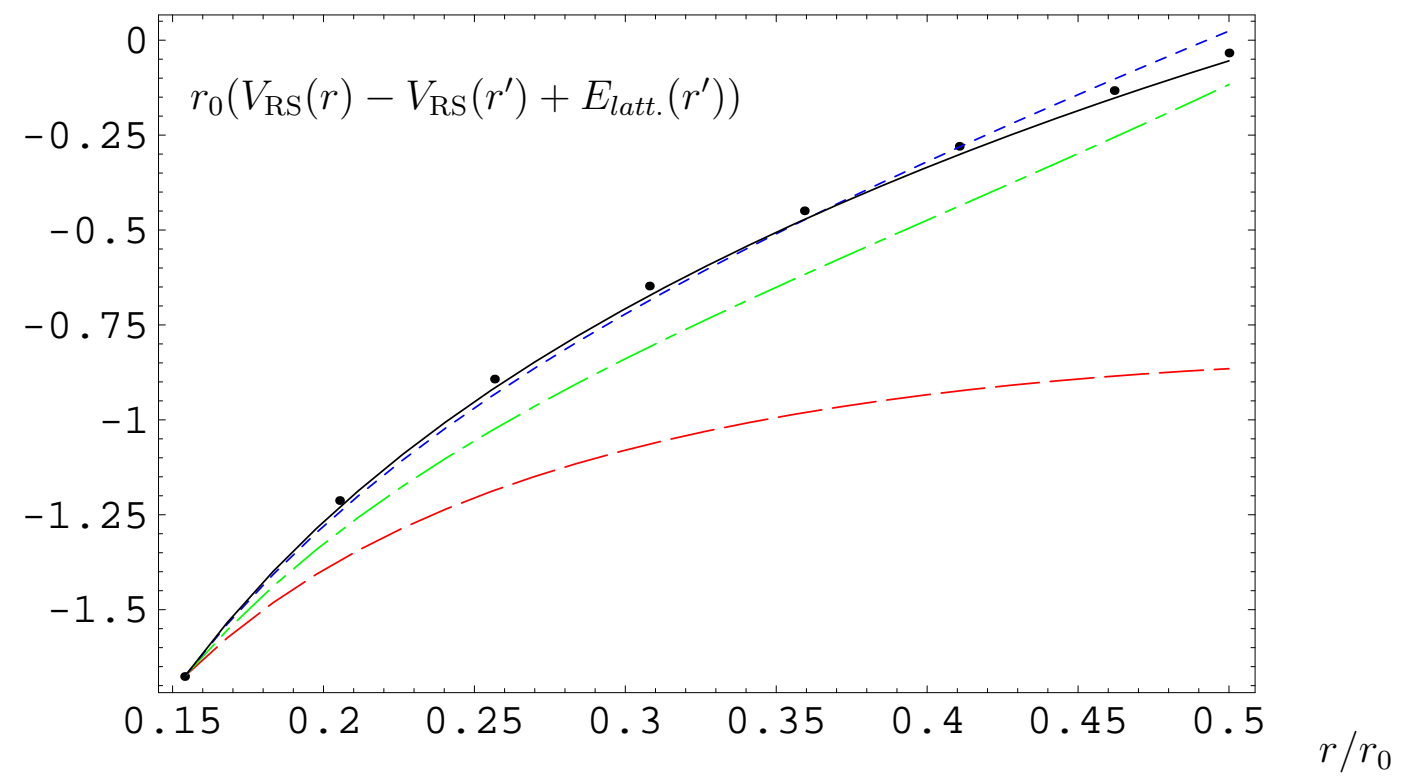

Figure 7: Plot of $r_{0}\left(V_{\mathrm{RS}}(r)-V_{\mathrm{RS}}\left(r^{\prime}\right)+E_{\text {latt. }}\left(r^{\prime}\right)\right)$ versus $r$ at tree (dashed line), one-loop (dash-dotted line), two-loops (dotted line) and three loops (estimate) plus the RG expression for the ultrasoft logs (solid line) compared with the lattice simulations [2] $E_{\text {latt. }}(r)$. For the scale of $\alpha_{\mathrm{s}}(\nu)$, we set $\nu=1 / r . \nu_{f}=\nu_{u s}=2.5 r_{0}^{-1}$ and $r^{\prime}=0.15399 r_{0}$.

following way:

$$
V(r)=\int_{r_{0}}^{r} d r^{\prime} F\left(r^{\prime}\right)+V\left(r_{0}\right)=\int_{r_{0}}^{r} d r^{\prime} \frac{d V\left(r^{\prime}\right)}{d r^{\prime}}+V\left(r_{0}\right) .
$$

As far as we end up in the potential, we should have the same problems (and solutions) than in the previous sections. The point is how Eq. (21) is implemented in practice. The force also has an expansion in $\alpha_{\mathrm{s}}$ :

$$
F(r)=C_{F} \frac{\alpha_{q q}(1 / r)}{r^{2}}=\frac{C_{F}}{r^{2}} \alpha_{\mathrm{s}}\left\{1+a_{q q}^{(1)} \alpha_{\mathrm{s}}+\cdots\right\} .
$$

In practice, the force is introduced in Eq. (21) order by order in $\alpha_{\mathrm{s}}$. If the expansion parameter is $\alpha_{\mathrm{s}}(\nu)$, the potential is reconstructed order by order in $\alpha_{\mathrm{s}}(\nu)$ and we are exactly in one of the situations considered in this and the previous sections (Fig. 3). If the expansion parameter in the force is $\alpha_{\mathrm{s}}(1 / r)$, as it was made in Refs. [16, 18], we are in a new situation. The introduction of $\alpha_{\mathrm{s}}(1 / r)$ in the integral in Eq. (21) produces and infinite series in $\alpha_{\mathrm{s}}(1 / r)$ and $\alpha_{\mathrm{s}}\left(1 / r_{0}\right)$. At this respect, it is not clear what the expansion parameter is and the systematics of this procedure. In any case, here, we are just interested to see whether the renormalon cancellation is achieved for these calculations. At this respect, the point is that the expansion parameter in the potential is not $\alpha_{\mathrm{s}}(r)$. From the computational point of view, all the powers in $\alpha_{\mathbf{s}}(1 / r)$ and $\alpha_{\mathbf{s}}\left(1 / r_{0}\right)$ are considered to be of the same order. If we consider the leading order, one loop, approximation we have $\alpha_{q q}=\alpha_{\mathrm{s}}$ and $\beta\left(\alpha_{q q}\right)=-\beta_{0} \alpha_{\mathrm{s}} /(2 \pi)$ and we obtain

$$
\int_{r_{0}}^{r} d r^{\prime} F\left(r^{\prime}\right)=-C_{F} \frac{\alpha_{\mathrm{S}}(1 / r)}{r}\left(\sum_{n=0}^{\infty} n !\left[\frac{\beta_{0} \alpha_{\mathrm{s}}(1 / r)}{2 \pi}\right]^{n}-\sum_{n=0}^{\infty} n !\left[\frac{\beta_{0} \alpha_{\mathrm{s}}\left(1 / r_{0}\right)}{2 \pi}\right]^{n}\right) .
$$


Both terms have the renormalon (in the large $\beta_{0}$ approximation) so that they cancel each other. We expect that higher orders computations will reconstruct the QCD renormalon but yet the renormalon cancellation should remain at any order since the same (partial) piece of the renormalon appears for $\int^{r} d r^{\prime} F^{n-l o o p s}\left(r^{\prime}\right)$ than for $\int^{r_{0}} d r^{\prime} F^{n-l o o p s}\left(r^{\prime}\right)$. This explains within a renormalon-dominance approach the convergence of computations using this framework even if no reference to renormalon was made for these calculations.

It is worth stressing that within this renormalon-based scheme calculation, we can now account for the logs correctly by setting $\nu=1 / r$ (see Fig. 7). On the other hand, we introduce some errors when fixing $\nu=1 / r$, since we are only able to perform an approximate computation of the renormalon, which do not exist in the calculation of the slope with $\nu=$ constant. The two things compete with each other in the final accuracy of the result (compare Figs. 3 and 7). Indeed, once the renormalon cancellation has been achieved, the resummation of logs does not appear to be very important. At least, if we do not choose our scale very far from the typical scales at study. If we go to scales smaller than $2.5 r_{0}^{-1}$, the calculation with $\nu=1 / r$ appears to give better results (compatible with lattice, although with larger errors, up to amazingly long distances $\left(\sim 0.8 r_{0}\right)$ ) than the calculation with fixed $\nu$ (see Fig. 8). This may signal that log resummation becomes important for these scales (note that we have chosen $\nu=1 / 0.15399 r_{0}^{-1}$ as the starting point of the log evolution, for a smaller value of $\nu$ an improvement may be obtained). It should be studied further whether perturbation theory can indeed describe lattice data in the regime $0.5-0.8 r_{0}$, since this would support the claims of Ref. [16] in this direction.
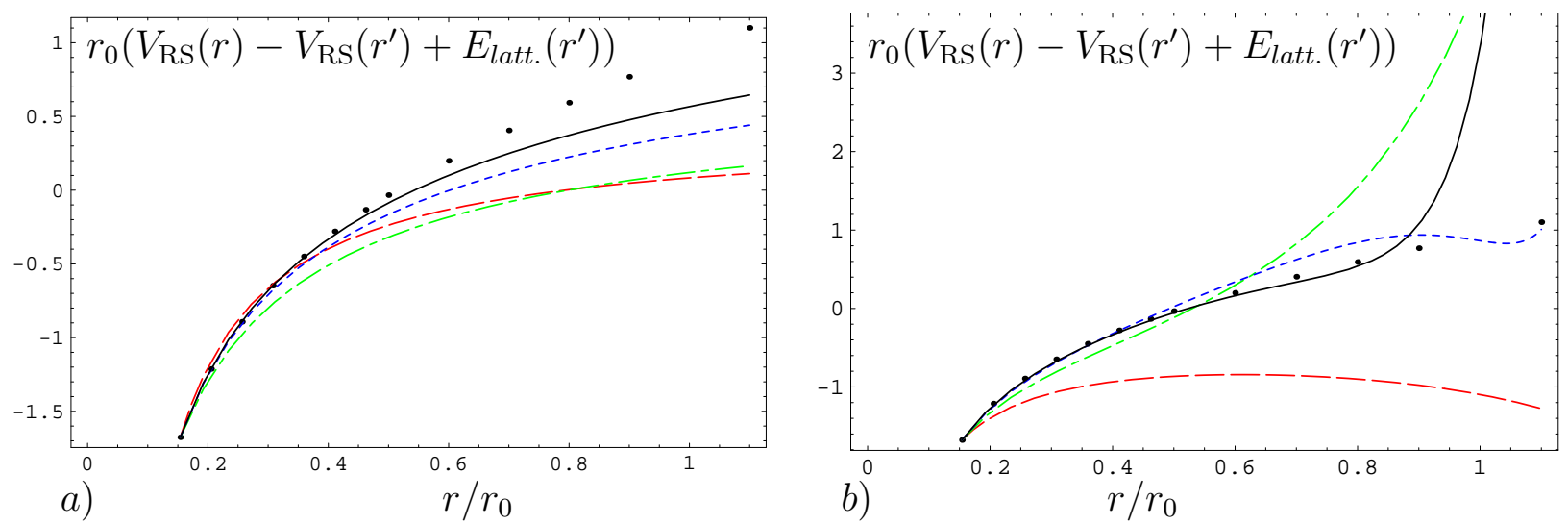

Figure 8: Figs. a) and b) correspond to Figs. 3 and 7 respectively up to the value $r=1.1 r_{0}$.

The main result of this section is to show that all the different results found in the previous and this section as well as in the literature about the convergence (or not convergence) of the perturbative series can be explained by the presence of the renormalon. We have also shown that, with a renormalon-based scheme, agreement with lattice is obtained. The point is that we now know the constant to be subtracted and its dependence in $\alpha_{\mathrm{s}}$, therefore, we can set $\alpha_{\mathrm{s}}$ at the same scale and obtain the cancellation for any $r$. In this way, we obtain the $\log$ resummation and the renormalon cancellation. One potential problem is that other $\operatorname{logs}\left(\ln \left(r \nu_{f}\right)\right)$ appear (in the physical, finite mass, case this problem does not really show up because one usually sets $\nu_{f} \lesssim 1 / r$ being $r$ the typical size of the system). 


\section{Bounds on short-distance non-perturbative poten- tials}

We now study possible non-perturbative effects in the static potential. The first thing to notice is that any non-perturbative effects should be small and compatible with zero since perturbation theory is able to explain lattice data within errors. We can make this statement more quantitative by using the lattice data obtained in Ref. [2] where the continuum limit has been reached. For these lattice points the systematic and statistic errors are very small (smaller than the size of the points). Therefore, the main sources of uncertainty of our (perturbative) evaluation come from the uncertainty in the value of $\Lambda_{\overline{\mathrm{MS}}}\left( \pm 0.48 r_{0}^{-1}\right)$ obtained from the lattice [23] and from the uncertainty in higher orders in perturbation theory. We show our results in Fig. 9. ${ }^{8}$ The inner band reflects the uncertainty in $\Lambda_{\overline{\mathrm{MS}}}$ whereas the outer band is meant to estimate the uncertainty due to higher orders in perturbation theory by allowing $c_{0}$ to change by \pm 146 . This is half its value according to the estimate in Table 1. This may seem a conservative variation if we take into account that the difference with the estimate obtained using Pade-approximats [22] is $\delta c_{0} \sim 20$.

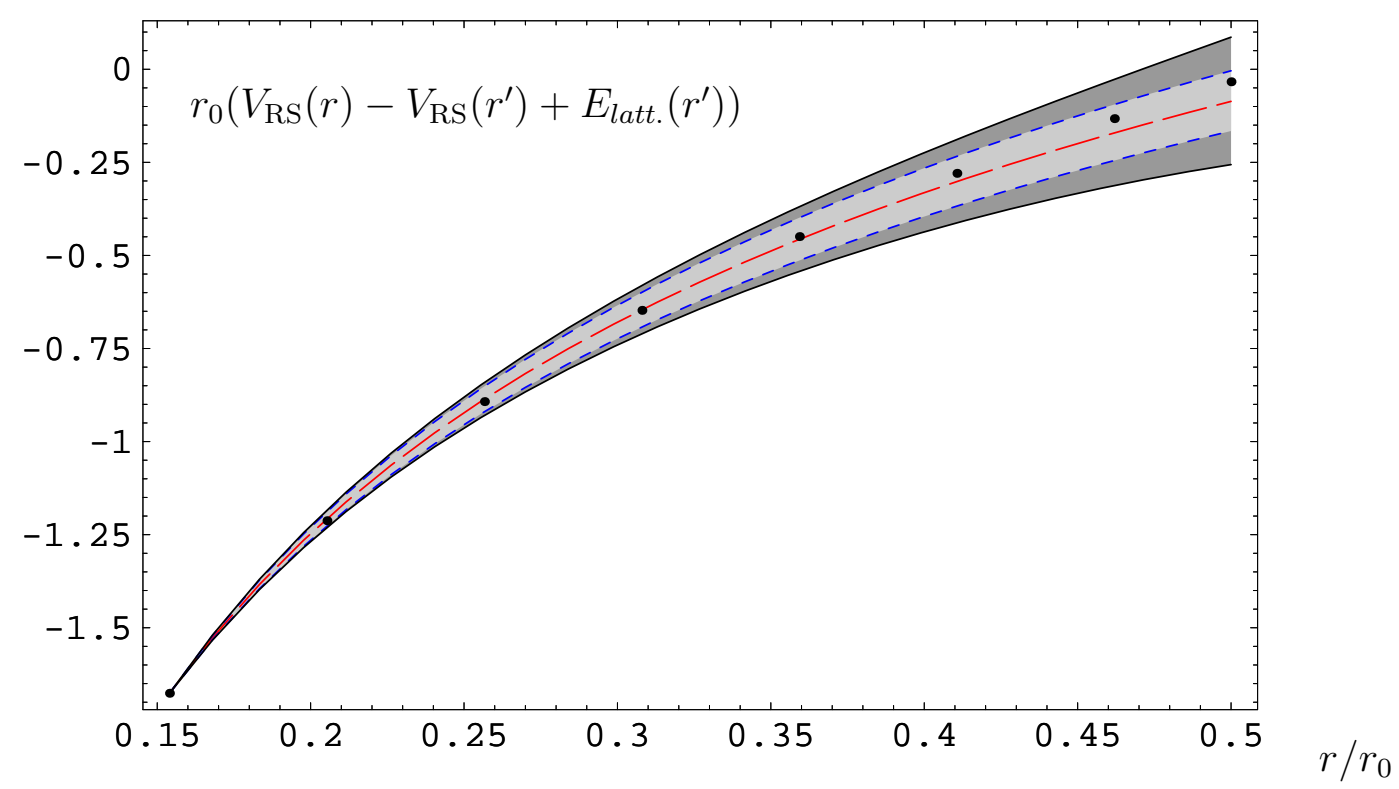

Figure 9: Plot of $r_{0}\left(V_{\mathrm{RS}}(r)-V_{\mathrm{RS}}\left(r^{\prime}\right)+E_{\text {latt. }}\left(r^{\prime}\right)\right)$ versus $r$ at three loops (estimate) plus the leading single ultrasoft log (dashed line) compared with the lattice simulations [2] $E_{\text {latt. }}(r)$. For the scale of $\alpha_{\mathrm{s}}(\nu)$, we set $\nu=1 / 0.15399 r_{0}^{-1} . \nu_{u s}=2.5 r_{0}^{-1}$ and $r^{\prime}=0.15399 r_{0}$. The inner and outer band are meant to estimate the errors in $\Lambda_{\overline{\mathrm{MS}}}$ and $c_{0}$. For further details see the main text.

\footnotetext{
${ }^{8}$ We have performed the estimation of the errors with the results of Fig. 3 ( $\nu=$ constant), since they do not introduce errors due to the evaluation of the renormalon. Nevertheless, a similar conclusion had been achieved if the analysis had been done with the results of Fig. $7(\nu=1 / r)$.
} 


\subsection{Bounds on a short-distance quadratic potential}

The non-perturbative effects should be encoded within Eq. (7). In general, this equation is a convolution of two scales: $\Lambda_{\mathrm{QCD}}$ and $\alpha_{\mathrm{s}} / r$. The explicit functionality is unknown since it involves the knowledge of the details of the non-perturbative dynamics. In the formal limit $1 / r \gg \Lambda_{\mathrm{QCD}} \gg \alpha_{\mathrm{s}} / r$, it is possible to know the scaling of the potential in $\Lambda_{\mathrm{QCD}}$ and $r$ based on dimensional arguments [27]:

$$
\delta E_{\mathrm{US}}\left(r, \nu_{u s}\right) \simeq \frac{T_{F}}{3 N_{c}} \mathbf{r}^{2} \int_{0}^{\infty} d t\left\langle g \mathbf{E}^{a}(t) \phi(t, 0)_{a b}^{\mathrm{adj}} g \mathbf{E}^{b}(0)\right\rangle\left(\nu_{u s}\right) .
$$

This $r^{2}$ behavior for the non-perturbative potential is expected on the basis of the OPE in that limit.

The fact that we are able to put bounds on our perturbative evaluation of the potential and that the errors of the lattice simulations are very small allows us to put bounds on the non-perturbative effects. This is of utmost importance since these non-perturbative effects produce the largest errors in the determination of the bottom mass using the $\Upsilon(1 S)$ mass. Unfortunately, even though lattice simulations of the static potential are very precise, we can not obtain strong constraints on the non-perturbative effects. The main source of error is due to the uncertainty in $\Lambda_{\overline{\mathrm{MS}}}$. Assuming a non-perturbative potential $V \sim A r^{2}$, we obtain

$$
|A| \lesssim 0.6 r_{0}^{-3}
$$

from the variation of $\Lambda \overline{\mathrm{MS}}$. If a potential $0.6 r_{0}^{-3} r^{2}$ were introduced as a correction in the binding energy of the $\Upsilon(1 S)$, it may induce a correction of $\sim 100 \mathrm{MeV}$ in the mass of the $\Upsilon(1 S)$ (however, let us also stress that lattice simulations are also compatible with $A=0$ ). Nevertheless, if lattice determinations of $\Lambda_{\overline{\mathrm{MS}}}$ could be improved, they would provide one of the most promising ways to control the errors in the determination of the bottom $\overline{\mathrm{MS}}$ mass using the $\Upsilon(1 S)$ mass. Let us also note that one can turn the problem around. If one assumes the non-perturbative effects to be small one can improve the lattice determination of $\Lambda_{\overline{\mathrm{MS}}}$ from the static potential data.

\subsection{Bounds on a short-distance linear potential}

The usual confining potential, $\delta V=\sigma r$, goes with an slope $\sigma=0.21 \mathrm{GeV}^{2}$. In lattice units we take: $\sigma=1.35 r_{0}^{-2}$. Can we discriminate such potential at short distances with the available lattice data? The answer is yes as we can see from Fig. $10 .{ }^{9}$ The introduction of a linear potential at short distances with such slope is not consistent with lattice simulations. This is even so after the errors considered in Fig. 9 have been included. This should not come as a surprise since this linear potential appears as an effect of long distance (not short distance) and it is not expected to appear from Eq. (7). Therefore, it follows that the use of the Cornell potential (with the perturbative static potential emanated from QCD instead of a pure $1 / r$ potential times a fitted constant) as a phenomenological fit of the static potential introduces systematic errors if the typical inverse Bohr radius scale of the heavy quarkonium

\footnotetext{
${ }^{9}$ We have used the results from Fig. 3, since they do not introduce errors due to the evaluation of the renormalon. Nevertheless, the same conclusion had been achieved with the results from Fig. 7.
} 
system to study lye in the short distance regime as it is the case, for instance, for the $\Upsilon(1 S)$.

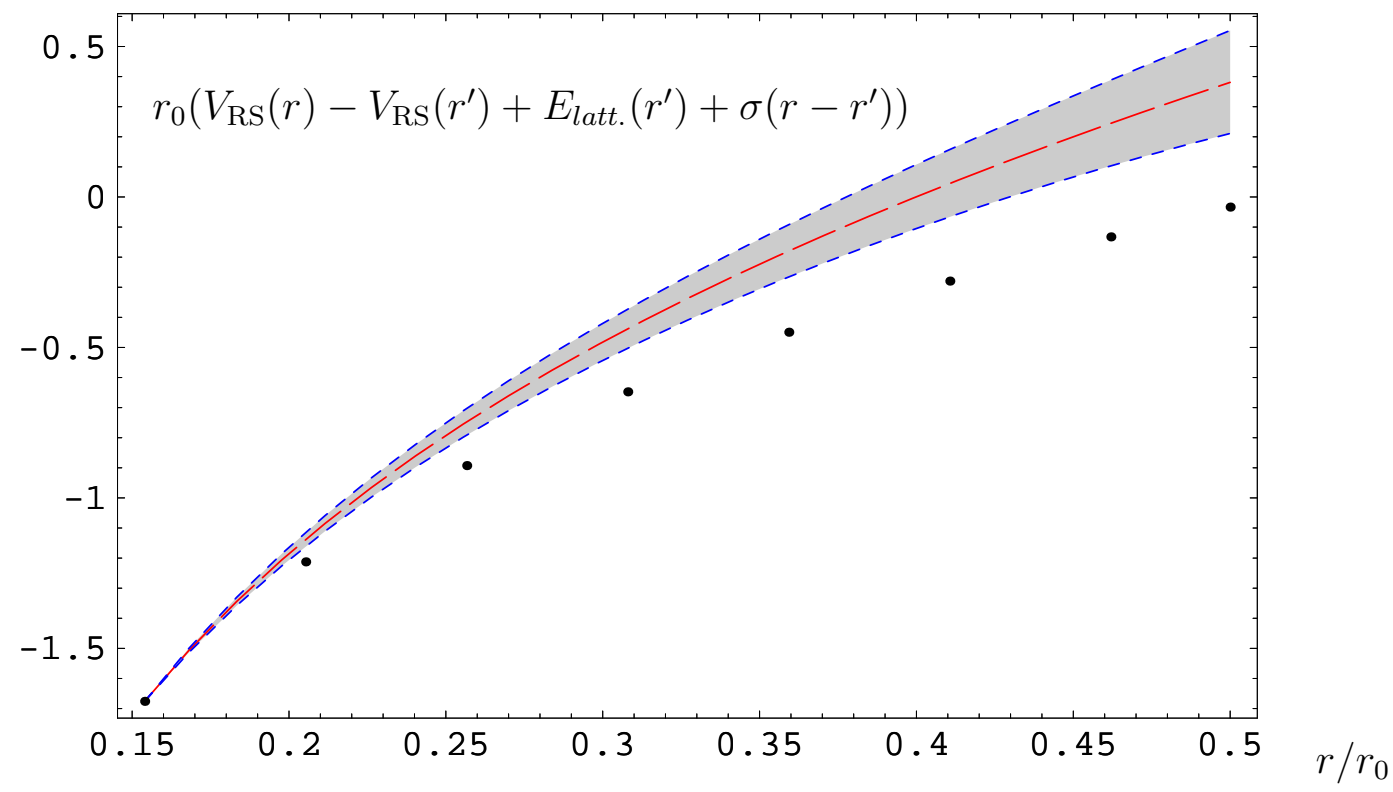

Figure 10: Plot of $r_{0}\left(V_{\mathrm{RS}}(r)-V_{\mathrm{RS}}\left(r^{\prime}\right)+E_{\text {latt. }}\left(r^{\prime}\right)+\sigma\left(r-r^{\prime}\right)\right)$ versus $r$ at three loops (estimate) plus the leading single ultrasoft log compared with the lattice simulations [2] $E_{\text {latt. }}(r)$. For the scale of $\alpha_{\mathrm{s}}(\nu)$, we set $\nu=1 / 0.15399 r_{0}^{-1}$. $\nu_{u s}=2.5 r_{0}^{-1}$ and $r^{\prime}=0.15399 r_{0}$. The dashed band is meant to estimate the combined error due $\Lambda_{\overline{\mathrm{MS}}}$ and $c_{0}$ (see Fig. 9).

On the other hand, recently, there have been claims about the possible existence of a linear potential at short distances $[13,14]$. Expected it to be of different physical origin than the long distance linear potential, it may have a different slope than the (long distance) static potential discussed above. It would be very important to discriminate its existence, since such behavior at short distances is at odds with the OPE. This is indeed possible, since the short-distance linear potential expected in Ref. $[13,14]$ have an slope of the order of magnitude of the long-distance confining potential that we have already ruled out above. Therefore, we can conclude that no linear potential exists at short distance (with the present estimates for its slope).

\section{Conclusions}

In this paper, we have shown (Figs. 3 and 7) that perturbation theory can explain lattice simulations of the static potential up to scales of order $\sim 2 r_{0}^{-1}$ once the renormalon has been taken into account without the need of non-perturbative effects. Quite remarkable, lattice simulations are precise enough to see the scaling log behavior predicted by asymptotic freedom and not just the pure $1 / r$ behavior of the Coulomb potential.

We have shown that the different results one may find in the literature as well as in sections 2 and 3 about the convergence (or not) of the perturbative series to the lattice 
result can be explained by the presence of the renormalon. In particular, we can explain why a calculation (Fig. 3) within perturbation theory can agree with lattice data, if the perturbative expansion is made with $\alpha_{\mathrm{s}}(\nu)$ with $\nu$ constant, without an explicit reference to the renormalon, but not if the expansion is made with $\alpha_{\mathrm{s}}(1 / r)$ (Fig. 4). This explanation is given in a renormalon-based approach in a unified way. Moreover, our method allows to set $\nu=1 / r$ and, therefore, resum the $\ln (r \nu) \operatorname{logs}$ besides of cancelling the renormalon (Fig. 7).

We have tried to find bounds on possible non-perturbative potentials at short distances. As a matter of principle, large non-perturbative effects are ruled out with the above analysis. In particular, they are compatible with zero (Fig. 9). We have shown (Fig. 10) that lattice data disfavours a linear confining potential at short distances with the usual slope $\left(\sigma=1.35 r_{0}^{-2}\right)$ assigned to the confining potential at long distances. For a possible nonperturbative $r^{2}$ potential, the main source of error comes from the one associated to the determination of $\Lambda_{\overline{\mathrm{MS}}}$ in the lattice (the error due to higher order in perturbation theory appears to be less important). Therefore, we can not put very precise constraints on it in order to reduce the non-perturbative error on the determinations of the bottom $\overline{\mathrm{MS}}$ mass from the $\Upsilon(1 S)$ mass. Nevertheless, lattice simulations remain as one of the most promising possibilities to constraint the size of the non-perturbative effects at short distances.

\section{Acknowledgments}

We thank G.S. Bali for sharing his lattice data with us.

\section{References}

[1] G.S. Bali and K. Schilling, Phys. Rev. D47 (1993) 661; G.S. Bali and K. Schilling, Int. J. Mod. Phys. C4 (1993) 1167; G.S. Bali, K. Schilling and A. Wachter, Phys. Rev. D56 (1997) 2566.

[2] S. Necco and R. Sommer, Nucl. Phys. B622, 328 (2002).

[3] K.J. Juge, J. Kuti and C.J. Morningstar, Nucl. Phys. (Proc. Suppl.) 83, 503 (2000).

[4] W. Fischler, Nucl. Phys. B129, 157 (1977); Y. Schröder, Phys. Lett. B447, 321 (1999); B.A. Kniehl, A.A. Penin, V.A. Smirnov and M. Steinhauser, Phys. Rev. D65, 091503 (2002); M. Peter, Phys. Rev. Lett. 78, 602 (1997).

[5] N. Brambilla, A. Pineda, J. Soto and A. Vairo, Phys. Rev. D60 (1999) 091502.

[6] B.A. Kniehl and A.A. Penin, Nucl. Phys. B563, 200 (1999).

[7] A. Pineda and J. Soto, Phys. Lett. B495, 323 (2000).

[8] A.H. Hoang, A.V. Manohar and I.W. Stewart, Phys. Rev. D64, 014033 (2001).

[9] A.A. Penin and M. Steinhauser, private communication.

[10] U. Aglietti and Z. Ligeti, Phys. Lett. B364, 75 (1995). 
[11] A. Pineda, PhD. thesis, U. Barcelona, January (1998); A.H. Hoang, M.C. Smith, T. Stelzer and S. Willenbrock, Phys. Rev. D59, 114014 (1999); M. Beneke, Phys. Lett. B434, 115 (1998).

[12] G.S. Bali, Phys. Lett. B460, 170 (1999).

[13] F.G. Gubarev, M.I. Polikarpov and V.I. Zakharov, Mod. Phys. Lett. A14, 2039 (1999).

[14] Yu.A. Simonov, JETP Lett. 69, 505 (1999).

[15] N. Brambilla, Y. Sumino and A. Vairo, Phys. Lett. B513, 381 (2001); Phys. Rev. D65, 034001 (2002).

[16] Y. Sumino, Phys. Rev. D65, 054003 (2002); S. Recksiegel and Y. Sumino, Phys. Rev. D65, 054018 (2002).

[17] A. Hoang, Z. Ligeti and A.V. Manohar, Phys. Rev. Lett. 82, 277 (1999).

[18] S. Necco and R. Sommer, Phys. Lett. B523, 135 (2001).

[19] P. Boyle and G.S. Bali, Nucl. Phys. B (Proc. Suppl.) 106, 811 (2002).

[20] A. Pineda, JHEP0106, 022 (2001).

[21] A. Pineda and J. Soto, Nucl. Phys. B (Proc. Suppl.) 64, 428 (1998); N. Brambilla, A. Pineda, J. Soto and A. Vairo, Nucl. Phys. B566, 275 (2000).

[22] F.A. Chishtie and V. Elias, Phys. Lett. B521, 434 (2001).

[23] ALPHA collaboration: S. Capitani, M. Lüscher, R. Sommer and H. Wittig, Nucl. Phys. B544, 669 (1999).

[24] M. Beneke, Phys. Lett. B344, 341 (1995).

[25] M. Beneke, Phys. Rep. 317, 1 (1999).

[26] T. Lee, Phys. Rev. D56, 1091 (1997); Phys. Lett. B462, 1 (1999).

[27] I.I. Balitsky, Nucl. Phys. B254, 166 (1985). 